\title{
Investigation on urinary and serum alpha klotho in dogs with chronic kidney disease
}

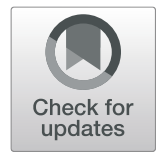

Hong jae $\mathrm{Yi}^{1}$, Jong bok Lee ${ }^{2}$, Kyu pil Lee ${ }^{1}$, Ye-In Oh${ }^{1}$, Kun ho Song ${ }^{1}$ and Kyoung won Seo ${ }^{{ }^{*}}$

\begin{abstract}
Background: As a co-receptor for fibroblast growth factor 23, klotho plays a pivotal role in phosphate metabolism. The kidney is known to be the main source of soluble alpha-klotho and the principal regulator of its concentration. Previous studies in human participants showed that the concentration of soluble alpha-klotho in serum and urine decreased in chronic kidney disease (CKD) patients. However, no previous study has assessed soluble alpha-klotho levels in dogs. This study aimed to measure serum and urinary alpha-klotho levels in CKD dogs and identify their associations with International Renal Interest Society (IRIS) CKD stages and other parameters known to be associated with CKD.

Results: Serum and urinary alpha klotho concentrations were measured by a commercially available canine-specific sandwich enzyme-linked immunosorbent assay kit and compared between groups by a nonparametric KruskalWallis test. Spearman's correlation coefficient was used to evaluate the relationships between variables. A stepwise multiple regression analysis was performed to estimate the effects of independent predictors on klotho concentrations. The urine klotho-to-creatinine ratio $(\mathrm{UrKl} / \mathrm{Cr}$ ) was significantly lower in stage 3 dogs than the control group and was significantly lower in dogs with stage 3 and 4 CKD than in those with stage 1 and 2 disease. UrKI/Cr was negatively correlated with serum symmetric dimethylarginine (sSDMA), blood urea nitrogen (BUN), creatinine, and phosphorus concentration. Serum alpha-klotho concentration in dogs with stages 2 and 3 CKD was significantly lower than those in the control group. There was no significant correlation between serum alphaklotho and BUN, creatinine, and phosphorus concentrations. No statistically significant differences were observed in UrKI/Cr and serum alpha-klotho concentration between groups based on sex, age, urine protein-to-creatinine ratio (UPC), or blood pressure.

Conclusions: UrKl/Cr decreased in dogs with advanced CKD, and it was negatively correlated with SSDMA, BUN, creatinine, and phosphorus concentrations. Thus, klotho is associated with CKD and its clinical consequences, including CKD-mineral bone disorder, in dogs. Although serum klotho concentration was negatively correlated with SSDMA levels, it was not apparently related to IRIS CKD stage or other parameters known to be associated with CKD.
\end{abstract}

Keywords:: Canine, Chronic kidney disease-mineral bone disorder (CKD-MBD), Klotho, SDMA, Serum, Urine

\footnotetext{
* Correspondence: kwseo@cnu.ac.kr

${ }^{1}$ VMTH of College of Veterinary Medicine, Chungnam National University,

N13-2, \#308, 99 Daehak-ro, Yuseong-gu, Daejeon, Republic of Korea

Full list of author information is available at the end of the article
}

(c) The Author(s). 2020 Open Access This article is licensed under a Creative Commons Attribution 4.0 International License, which permits use, sharing, adaptation, distribution and reproduction in any medium or format, as long as you give appropriate credit to the original author(s) and the source, provide a link to the Creative Commons licence, and indicate if changes were made. The images or other third party material in this article are included in the article's Creative Commons licence, unless indicated otherwise in a credit line to the material. If material is not included in the article's Creative Commons licence and your intended use is not permitted by statutory regulation or exceeds the permitted use, you will need to obtain permission directly from the copyright holder. To view a copy of this licence, visit http://creativecommons.org/licenses/by/4.0/. The Creative Commons Public Domain Dedication waiver (http://creativecommons.org/publicdomain/zero/1.0/) applies to the data made available in this article, unless otherwise stated in a credit line to the data. 


\section{Background}

Chronic kidney disease (CKD) is one of the most common kidney diseases in dogs. The prevalence of CKD in dogs was estimated to be $1.5 \%$ in general practice [1]. Reduced glomerular filtration rate (GFR) can decrease phosphorus excretion and result in hyperphosphatemia. Renal secondary hyperparathyroidism (RSHP), which is generally believed to be the clinical consequence of hyperphosphatemia, is reported in around $76 \%$ of CKD dogs, and in over $96 \%$ of dogs with over International Renal Interest Society (IRIS) stage 3 and over of the disease $[2,3]$. The term "chronic kidney disease-mineral bone disorder" (CKD-MBD) has been used in humans and animals to describe a condition characterized by renal osteodystrophy and abnormal mineral metabolism with high levels of serum phosphate, fibroblast growth factor (FGF)-23, and parathyroid hormone $(\mathrm{PTH})$ [4-6]. Klotho deficiency was suggested to be associated with or even induce these abnormal metabolic consequences [7-11].

The klotho gene was accidentally discovered in mice in 1997 [12]. Two forms of klotho protein, membranous and soluble, are consistently detected in vivo. The membranous form is mainly expressed on the proximal and distal tubules of the kidney [12-14]. Interactions of FGF-23 and the FGF receptor and subsequent signaling require binding of membranous klotho to FGF receptors and formation of the klothoFGF receptor complex, which binds to FGF-23 with much higher affinity than klotho or the FGF receptor alone [15-17]. Under physiological conditions, FGF23 suppresses PTH and calcitriol secretion to reduce serum phosphorus concentrations. In CKD, decreased expression of membrane-bound klotho limits FGF-23mediated signal transduction through FGF receptorklotho complexes, resulting in an increase in parathyroid hormone levels [5]. Moreover, soluble klotho has been reported to directly control phosphorus excretion in the kidney and participate in systemic mineral homeostasis by regulating $1 \alpha$-hydroxylase activity and PTH and FGF-23 secretion [18-20].

Soluble alpha-klotho is generated when the extracellular domain of membranous alpha-klotho is cleaved and released into plasma [21-24]. Soluble alpha-klotho is a major functional form in the circulation $[25,26]$ and is also found in cerebrospinal fluid and urine [27-29]. The kidney is known to be the main source of soluble alphaklotho and the principal regulator of its concentration $[24,30,31]$. Therefore, it seems reasonable to assume that nephron loss can lead to reduced production and release of soluble klotho. Significantly reduced levels of renal alpha-klotho mRNA were observed in several studies with rodent CKD models [31, 32]. Human studies have demonstrated that the soluble alpha-klotho concentration in serum and urine is decreased in CKD patients [27, 33] and that the serum alpha-klotho level tends to reduce as the CKD stage advances [34]. However, in contrast, some studies have reported no change in klotho concentration depending on the kidney function [35-37].

Recently, one study confirmed that the concentration of plasma FGF-23 increases as CKD advances and is significantly different between IRIS stages 1 and 2 versus stages 3 and 4 in dogs [38]. However, to the authors' knowledge, no previous study has assessed soluble alpha-klotho, which plays a key role with FGF-23 in CKD-MBD in dogs. Therefore, the aim of this study was to identify the characteristics of soluble alpha-klotho in CKD dogs by measuring serum and urinary alpha-klotho levels and verify their association with IRIS CKD stages and other parameters, including serum SDMA (sSDMA), creatinine, blood urea nitrogen (BUN), and phosphorus concentrations, which are known to be associated with CKD.

\section{Results}

The median age of dogs in the CKD group and control group were significantly different $(p<0.001)$. The results of laboratory analysis measuring the urine klotho-tocreatinine ratio $(\mathrm{UrKl} / \mathrm{Cr})$ and the serum alpha-klotho, sSDMA, BUN, creatinine, and phosphorus levels are presented in Table 1. The soluble alpha-klotho analysis was performed with serum and urine samples. Calculated $\mathrm{UrKl} / \mathrm{Cr}$ was used to minimize the effect of urine specific gravity. The median $\mathrm{UrKl} / \mathrm{Cr}$ (range) for all CKD dogs was lower $(5.85 \mathrm{pg} / \mathrm{gCr}, 0.08$ to 82.28$)$ than that of control dogs (13.16 pg/gCr, 5.75 to 34.84). UrKl/ $\mathrm{Cr}$ was significantly lower in stage 3 dogs than the control group $(p=0.001)$ and stage 1 CKD group $(p=$ 0.003; Fig. 1). Serum alpha-klotho concentration was significantly lower in dogs with stage $2(p=0.001)$ and 3 $(p=0.001)$ CKD in comparison with the control group. $\mathrm{UrKl} / \mathrm{Cr}$ in dogs with stage 3 and 4 CKD was significantly lower than that in dogs with stages 1 and 2 CKD $(p<$ $0.001)$. UrKl/Cr was negatively correlated with sSDMA $(r$ $=-0.661, p<0.001$; Fig. 2$)$, BUN $(r=-0.631, p<0.001$; Fig. 2), creatinine $(r=-0.651, p<0.001$; Fig. 2$)$, and phosphorus ( $r=-0.449, p=0.005$; Fig. 2 ) concentrations.

The median(range) serum alpha-klotho level in all CKD dogs was lower $(59.13 \mathrm{pg} / \mathrm{mL}, 0.47$ to $189.13 \mathrm{pg} /$ $\mathrm{mL})$ than that in control dogs $(132.25 \mathrm{pg} / \mathrm{mL}, 57.46$ to169.33 pg/mL; Table 1). Serum alpha-klotho concentration was negativelycorrelated with sSDMA level $(r=$ $-0.348, p=0.035$; Figure 3). There was no significant correlation between serum alpha-klothoconcentration and BUN $(p=0.297)$, creatinine $(p=0.061)$, andphosphorus $(p=0.693)$ concentration. There was no statisticallysignificant difference in $\mathrm{UrKl} / \mathrm{Cr}$ and serum alpha 
Table 1 Comparison of laboratory variables in control and chronic kidney disease dogs

\begin{tabular}{|c|c|c|c|c|c|c|}
\hline Laboratory Variable & $\begin{array}{l}\text { Control } \\
(n=10)\end{array}$ & $\begin{array}{l}\text { All CKD } \\
(n=27)\end{array}$ & $\begin{array}{l}\text { Stage } 1 \\
(n=8)\end{array}$ & $\begin{array}{l}\text { Stage } 2 \\
(n=8)\end{array}$ & $\begin{array}{l}\text { Stage } 3 \\
(n=7)\end{array}$ & $\begin{array}{l}\text { Stage } 4 \\
(n=4)\end{array}$ \\
\hline $\begin{array}{l}\text { UrKl/Cr } \\
(\mathrm{pg} / \mathrm{gCr})\end{array}$ & $\begin{array}{l}13.16 \\
(5.75-34.84)\end{array}$ & $\begin{array}{l}5.85 \\
(0.08-82.28)\end{array}$ & $\begin{array}{l}12.32 \\
(3.03-82.28)\end{array}$ & $\begin{array}{l}13.57 \\
(2.15-18.00)\end{array}$ & $\begin{array}{l}0.81 \\
(0.30-4.37)\end{array}$ & $\begin{array}{l}3.98 \\
(0.08-5.85)\end{array}$ \\
\hline $\begin{array}{l}\text { Serum Klotho } \\
(\mathrm{pg} / \mathrm{mL})\end{array}$ & $\begin{array}{l}132.25 \\
(57.46-169.33)\end{array}$ & $\begin{array}{l}59.13 \\
(0.47-189.13)\end{array}$ & $\begin{array}{l}78.32 \\
(10.47-122.47)\end{array}$ & $\begin{array}{l}30.14 \\
(0.47-72.47)\end{array}$ & $\begin{array}{l}19.8 \\
(2.47-70.47)\end{array}$ & $\begin{array}{l}127.47 \\
(77.13-189.13)\end{array}$ \\
\hline $\begin{array}{l}\text { SDMA } \\
(\mu \mathrm{g} / \mathrm{dL})\end{array}$ & $\begin{array}{l}8 \\
(5-13)\end{array}$ & $\begin{array}{l}28 \\
(8-74)\end{array}$ & $\begin{array}{l}13.5 \\
(8-17)\end{array}$ & $\begin{array}{l}22.5 \\
(18-31)\end{array}$ & $\begin{array}{l}37 \\
(34-47)\end{array}$ & $\begin{array}{l}58.5 \\
(52-74)\end{array}$ \\
\hline $\begin{array}{l}\text { Creatinine } \\
(\mathrm{mg} / \mathrm{dL})\end{array}$ & $\begin{array}{l}0.8 \\
(0.6-1)\end{array}$ & $\begin{array}{l}1.9 \\
(0.7-11.1)\end{array}$ & $\begin{array}{l}1.1 \\
(0.7-1.3)\end{array}$ & $\begin{array}{l}1.75 \\
(1.5-2.3)\end{array}$ & $\begin{array}{l}3 \\
(1.8-4.2)\end{array}$ & $\begin{array}{l}5 \\
(3.8-11.1)\end{array}$ \\
\hline $\begin{array}{l}\text { BUN } \\
(\mathrm{mg} / \mathrm{dL})\end{array}$ & $\begin{array}{l}21.4 \\
(9-33.8)\end{array}$ & $\begin{array}{l}55.7 \\
(6.8-167.7)\end{array}$ & $\begin{array}{l}25.05 \\
(6.8-47.5)\end{array}$ & $\begin{array}{l}51.25 \\
(19-108.2)\end{array}$ & $\begin{array}{l}89 \\
(43.4-111.8)\end{array}$ & $\begin{array}{l}135.6 \\
(76.7-167.7)\end{array}$ \\
\hline $\begin{array}{l}\text { Phosphorus } \\
\text { (mg/dL) }\end{array}$ & $\begin{array}{l}3.7 \\
(3.1-5.2)\end{array}$ & $\begin{array}{l}3.9 \\
(2.9-19)\end{array}$ & $\begin{array}{l}3.3 \\
(2.9-3.8)\end{array}$ & $\begin{array}{l}4 \\
(2.9-5.3)\end{array}$ & $\begin{array}{l}5.9 \\
(3.1-8.38)\end{array}$ & $\begin{array}{l}11.15 \\
(4.39-19)\end{array}$ \\
\hline
\end{tabular}

Results are presented as median and range.

$\mathrm{UrKI} / \mathrm{Cr}$, urine klotho-to-creatinine ratio; SDMA, symmetric dimethylarginine; BUN, blood urea nitrogen.

klothoconcentration between different groups based on sex, age, urineprotein-to-creatinine ratio (UPC), or blood pressure (Figure 4).

Stepwise multiple regressionanalysis was performed with sSDMA, BUN, creatinine, and phosphorusconcentrations to predict $\mathrm{UrKl} / \mathrm{Cr}$. Of these variables, only sSDMA concentration $(B=-0.479)$ wasidentified as a predictive variable (total $\mathrm{R}^{2}=0.229, p=0.003$ ).

\section{Discussion}

To the authors' knowledge, this is the first study assessing soluble klotho in dogs. In the current study, the characteristics of serum and urinary klotho were investigated in CKD dogs. UrKl/Cr was significantly lower in stage 3 CKD group in comparison with those in the control and stage 1 groups. In addition, $\mathrm{UrKl} / \mathrm{Cr}$ of stage 3 and stage 4 CKD dogs was significantly lower than that of stage 1 and stage 2 CKD dogs. Median $\mathrm{UrKl} / \mathrm{Cr}$ in the stage 4 CKD group was lower than that in the early stages, although it was not significantly different from that in the control group. Moreover, $\mathrm{UrKl} / \mathrm{Cr}$ was inversely correlated with all tested parameters, including sSDMA, creatinine, BUN, and phosphorus levels, in CKD dogs. In addition, stepwise multiple regression analysis showed that SSDMA was an independent predictor of $\mathrm{UrKl} / \mathrm{Cr}$. The significantly lower $\mathrm{UrKl} / \mathrm{Cr}$ in dogs with progressed CKD and its correlation with various parameters reflecting either kidney function or the severity of kidney disease suggests that klotho is associated with CKD and the development of its clinical consequences in dogs.

In a previous study on dogs with CKD, increased levels of FGF-23 and PTH were confirmed in IRIS CKD stage 3 and 4 dogs [38]. However, measurable concentrations of klotho could not be detected in that study using the alpha-klotho enzyme-linked immunosorbent assay (ELIS
A) kit of IBL-America (Minneapolis, USA), which was the only available ELISA kit for detecting human or mouse klotho protein. The alpha-klotho protein sequence is known to be $88 \%$ identical between humans and dogs and $98 \%$ identical between humans and mice [39]. Since using the ELISA kit manufactured especially for canine tests was expected to enhance the accuracy of the test, the ELISA kit measuring canine-specific soluble alpha klotho was used in this study. Additionally, serum and urine samples were never thawed before ELISA assay because a recent study showed that the ELISA value of serum klotho decreases as samples undergo repeated freeze-thaw cycles [40].

Notably, the UrKl/Cr of dogs with advanced CKD was significantly reduced and correlated with various parameters, while serum alpha-klotho did not show these correlations in our study. This finding suggests that urinary klotho is more associated with kidney function or damage than serum klotho. It is also consistent with the reported characteristics of soluble klotho in CKD patients, which indicated that the amount of urinary klotho, rather than serum klotho, are linked to the extent of nephron function [27]. In this study, the amount of urinary klotho excreted for $24 \mathrm{~h}$ trended towards lower values as the CKD stage advanced, whereas serum klotho levels did not. The correlations with various parameters related to renal function and significant differences between advanced stages and early stages suggest that $\mathrm{UrKl} / \mathrm{Cr}$ has potential as a biomarker of kidney disease or can be used when monitoring disease progression. A possible advantage of using urinary klotho as a diagnostic or monitoring tool is that it is noninvasive in comparison with existing tools requiring venipuncture. Further studies are needed to confirm its usefulness as a biomarker of kidney disease. 

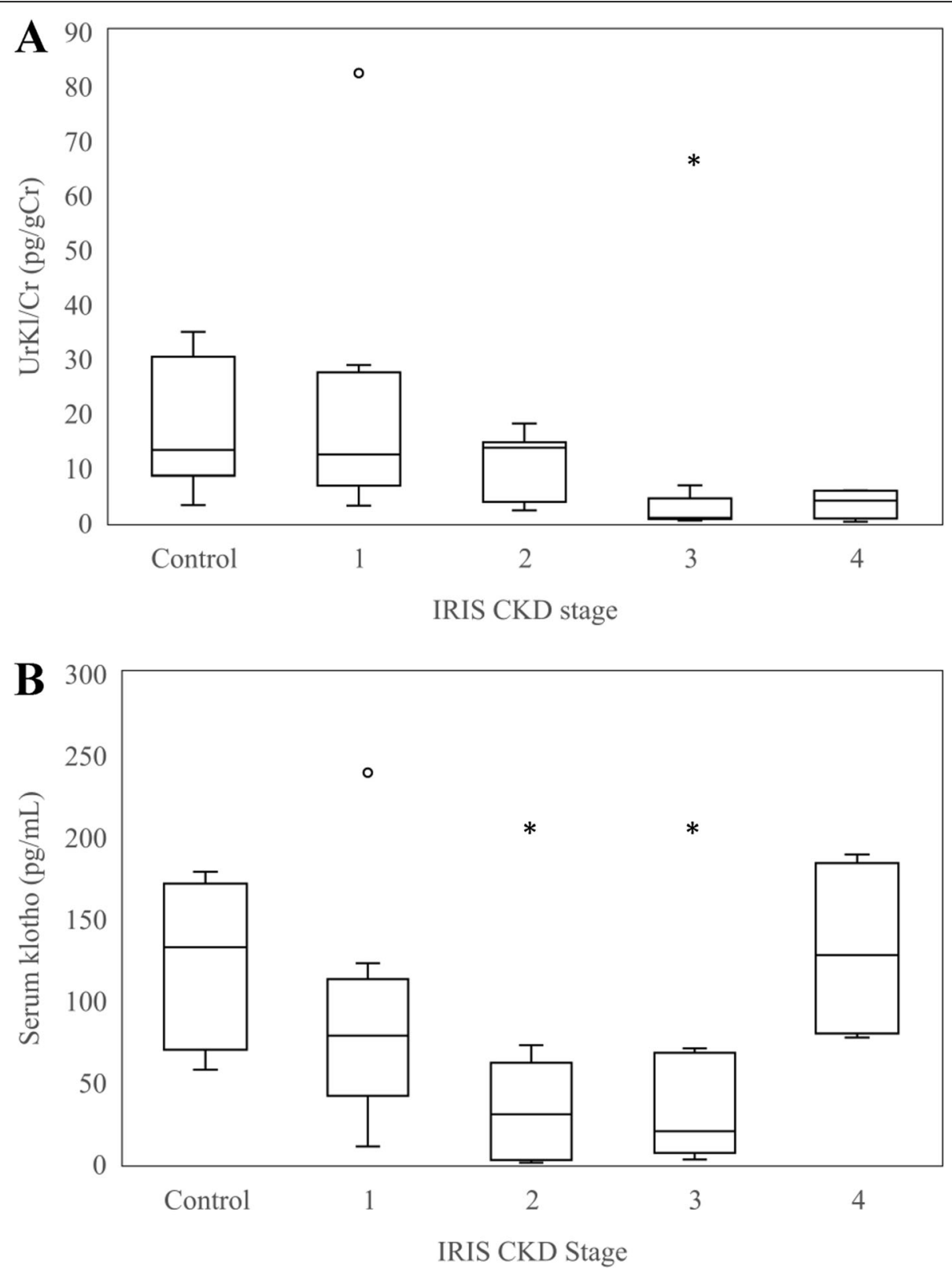

Fig. 1 Box-and-whisker plots of urinary (a) and serum (b) klotho concentrations. The boxes represent the 25th and 75thpercentiles, and the central lines in the boxes represent the median values.The whiskers represent the range of concentrations. Dots represent outliers. a The asterisk represents a statistically lower UrKI/Cr in dogs with IRISstages $3(p=0.001)$ compared to control dogs. b Asterisk represents astatistically lower serum klotho concentration in dogs with IRIS stages $2(p=0.001)$ and $3(p=0.001)$ compared to control dogs.

Contrary to UrKl/Cr, serum alpha-klotho concentration in stage 4 CKD dogs was higher than that in stage 3 CKD dogs, although the concentrations in stage 2 and stage 3 CKD dogs were significantly lower than those in control dogs. Interestingly, the relationship between serum klotho and sSDMA was better explained by a quadratic model $\left(p<0.001, \mathrm{R}^{2}=0.407\right)$ than a linear model $\left(p=0.428, \mathrm{R}^{2}=0.018\right)$. To date, no published studies of klotho have suggested a biological explanation related to this phenomenon. Further study is needed to validate this phenomenon and clarify the biological mechanisms underpinning it. Among the parameters examined, only sSDMA was negatively associated with serum alpha-klotho concentration. These results seem to correspond with the findings of human studies showing that plasma levels of serum klotho were not related to kidney function [37]. In a large cohort study of 312 patients, plasma klotho levels did not differ across CKD 

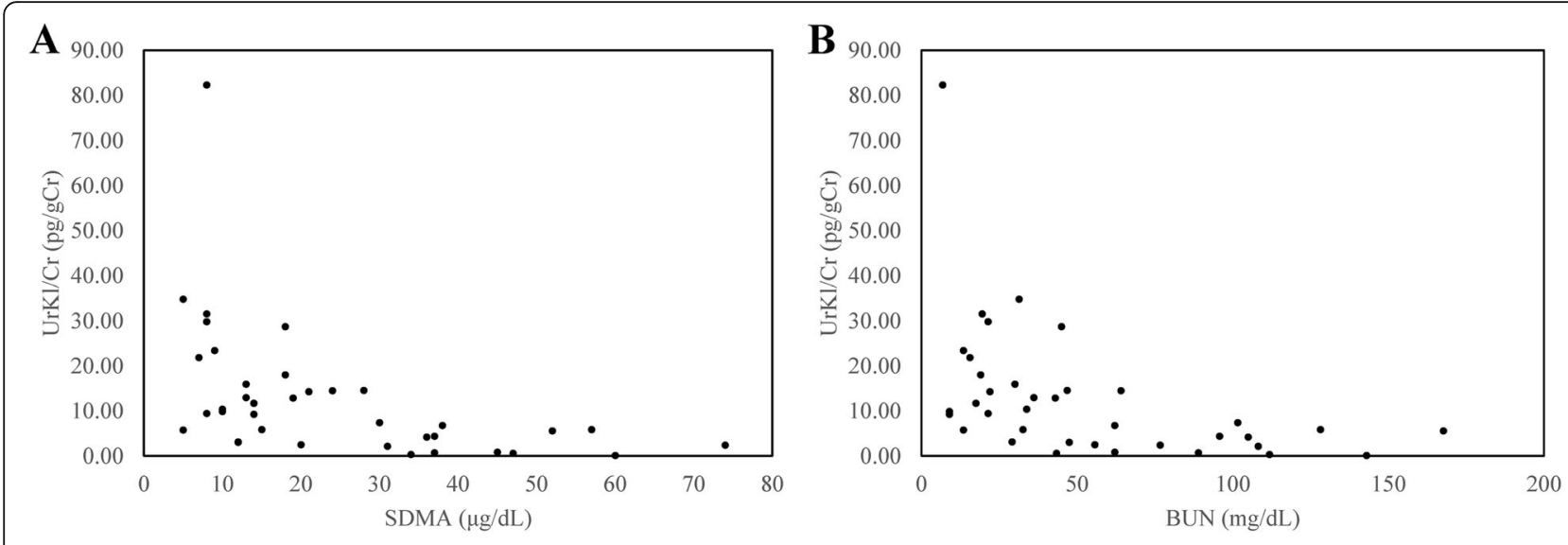

C

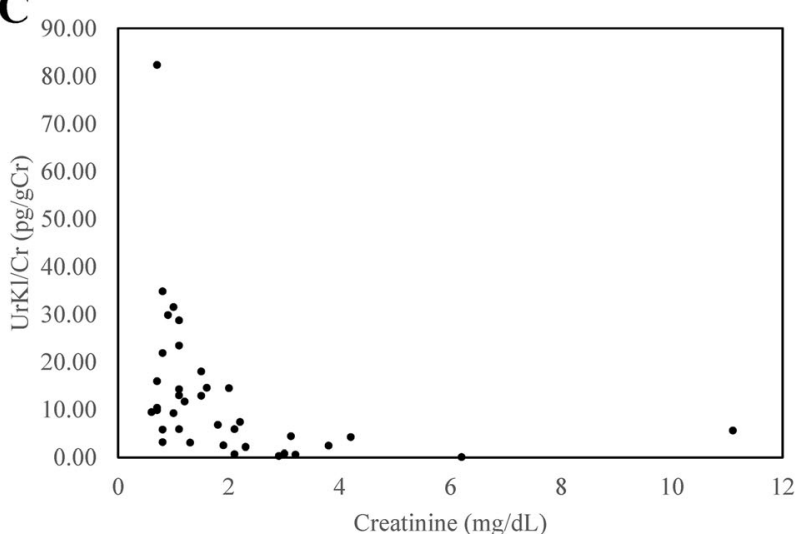

D

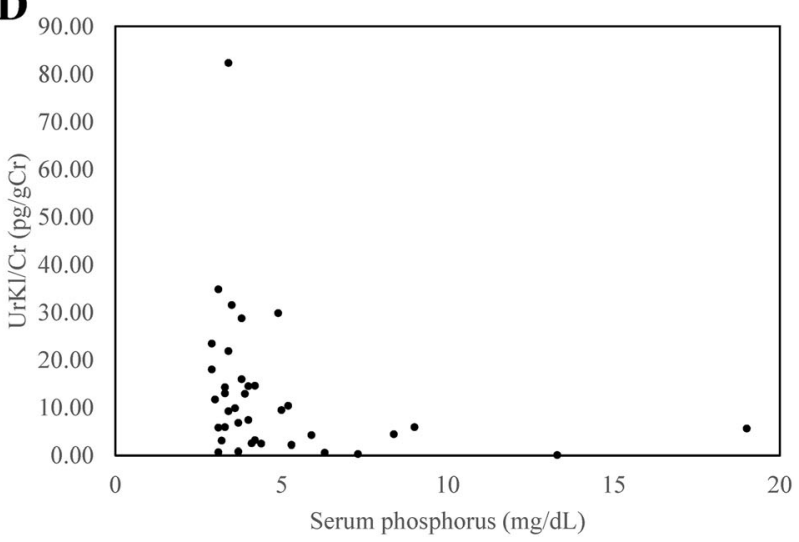

Fig. 2 Relationships between UrKI/Cr and a serum SDMA, b BUN, c creatinine, andd phosphorus concentrations. UrKI/Crwas negatively correlated with sSDMA ( $r=-0.661, p<0.001)$,BUN $(r=-0.631, p<0.001)$, creatinine $(r=-0.651, p<0.001)$ and phosphorus $(r=-0.449, p=0.005)$ concentrations.Spearman's correlation coefficient was used to evaluate the relationshipsbetween variables.

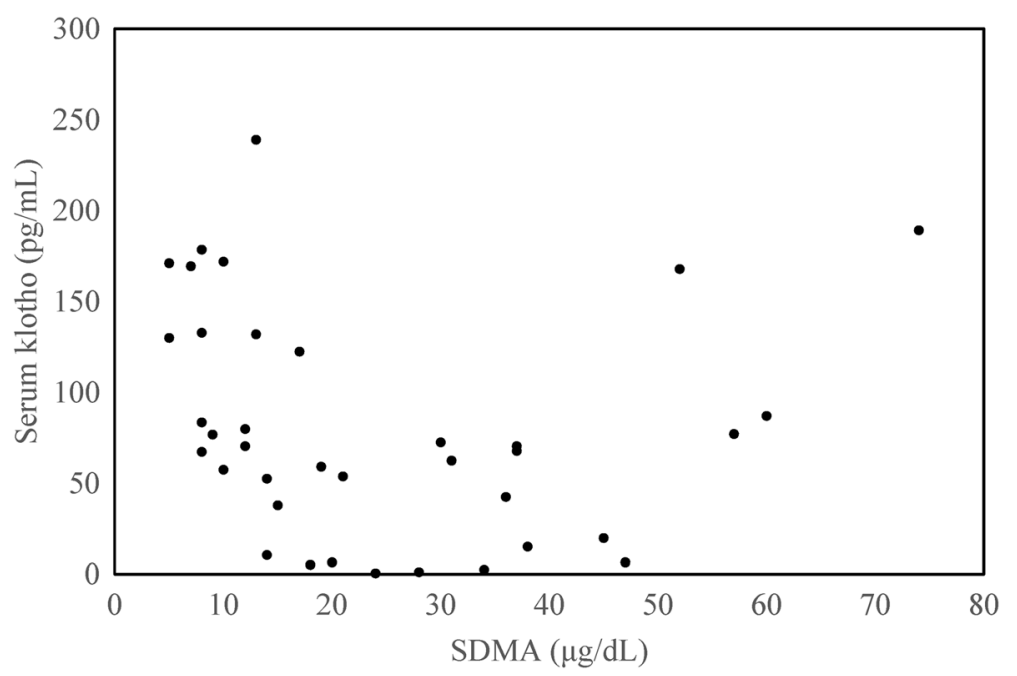

Fig. 3 Relationship between serum alpha-klotho andsSDMA concentrations. Serum alpha klotho was negatively correlated with sSDMAconcentration $(r=-0.348, p=0.035)$. 

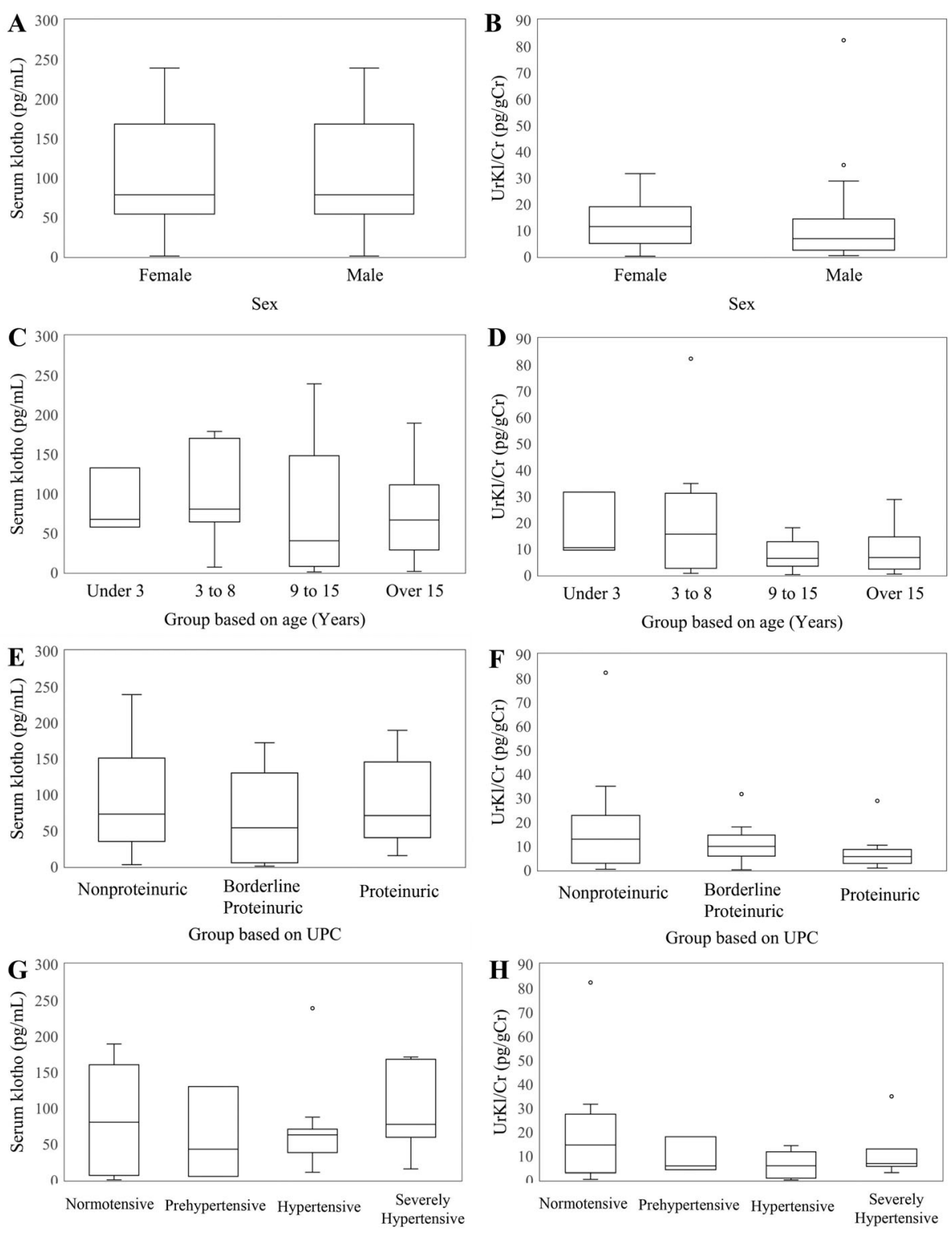

Group based on blood pressure

Group based on blood pressure

Fig. 4 Box-and-whisker plots illustrating klothoconcentrations based on sex, age, UPC, and blood pressure. The boxes representthe 25th and 75th percentiles, and the central lines in the boxes represent themedian values. The whiskers represent the range of concentrations. Dotsrepresent outliers. Serum klotho and sex (a),age (c), UPC (e), and blood pressure (g).Urinary klotho-to-creatinine ratio (UrKI/Cr) and sex (b), age (d), UPC (f), and blood pressure (h).

stages and were not significantly associated with GFR or other parameters of calcium-phosphate metabolism, which contradicted previous studies in which serum alpha-klotho concentration was associated with estimated GFR (eGFR) and was significantly decreased in CKD patients [33, 34]. In addition to this cohort study, research covering validation of immunoassays for soluble klotho showed that its concentration in CKD patients was higher than that in healthy controls [35]. These discordant results among studies on soluble alpha-klotho concentrations have been identified as a potential limitation restricting it from being used as biomarker of CKD. One possible reason for this discrepancy is the lack of a standardized assay to measure soluble klotho [41]. A previous human study evaluating three different commercial soluble klotho ELISA kits revealed poor inter- and intra-assay agreement between the ELISAs, implying that the problem may be not confined to just one ELISA manufacturer [42]. One recent study compared a commonly used commercial ELISA with 
immunoprecipitation-immunoblot (IP-IB) using stored serum samples of CKD patients [43]. IP-IB was strongly correlated with eGFR, whereas the commercial ELISA was not. Moreover, the IP-IB assay showed significant differences in serum klotho levels among stage 3 CKD, acute kidney injury, and end-stage renal disease groups. However, commercial ELISA failed to demonstrate differences across these groups. Therefore, this study concluded that the IP-IB assay shows better performance than the available commercial ELISA and recommended the IP-IB assay for measuring soluble klotho until a superior ELISA is developed [43]. Nevertheless, the laborintensive and time-consuming nature of the IP-IB assay limits its applicability. Besides, this assay may be operator-dependent because it requires meticulous quality control procedures, since it involves synthetic antibody production.

Our study had some limitations. First, the expression of klotho genes is known to be related to age, and a decrease in soluble alpha klotho concentration has been observed in aged people [44]. However, no previous study has assessed the effect of age on soluble klotho levels in dogs. Even though serum and urinary klotho levels were not significantly different between groups divided by age, we cannot rule out the possible effect of age on klotho concentrations in our study. To verify this effect, further studies on klotho concentration with larger numbers of healthy dogs of variable ages are needed. Second, we cannot exclude discordances among klotho tissue expression, plasma klotho levels, and klotho urinary excretion, although plasma klotho was suggested to reflect membrane-bound klotho. Quantification of the membranous klotho protein on the target cells of FGF23 would require invasive diagnostic procedures on target organs such as the kidney and parathyroid gland, which cannot be easily performed in clinical practice. Membranous klotho is cleaved and released into circulation, producing soluble klotho which is more readily accessible for measurement. However, the current methods for soluble klotho analysis may not be able to differentiate between full-length soluble klotho produced from cleavage of the membranous form [21-23] and further cleaved Kl1 and Kl2 klotho fragments. Further studies addressing the associations between different forms of klotho will improve our understanding of klotho in dogs with CKD.

\section{Conclusions}

$\mathrm{UrKl} / \mathrm{Cr}$ was decreased in dogs with advanced CKD. Furthermore, it showed a negative correlation to sSDMA, BUN, creatinine and phosphorus concentrations. These findings support the possibility that klotho is associated with CKD and its clinical consequences, including CKD-MBD, in dogs. Thus, we suggest that
$\mathrm{UrKl} / \mathrm{Cr}$ has the potential to serve as a biomarker, although further studies are needed to confirm its usefulness. Although serum klotho concentration was negatively correlated with sSDMA, it was not apparently related to IRIS CKD stage or other parameters known to be associated with CKD.

\section{Methods}

\section{Animal selection and allocation}

Client-owned dogs presenting to the veterinary teaching hospital of Chungnam National University and another referral clinic from April to October 2019 were included in this study. By the definition of CKD based on IRIS guidelines, dogs were diagnosed with CKD when they showed repeated minimally concentrated urine (urine specific gravity $<1.030)$ and met one of the following criteria: (1) azotemia based on serum creatinine (creatinine $\geq 1.4 \mathrm{mg} / \mathrm{dL}$ ); (2) high sSDMA concentration ( $\geq$ $18 \mu \mathrm{g} / \mathrm{dL}$ ); (3) ultrasonographic findings suggesting CKD (small, irregular kidneys or loss of corticomedullary junction). A control group comprised healthy dogs without clinical signs associated with urinary tract diseases, normal physical examination findings, normal complete blood count, and serum biochemistry profile results and urinalysis.

Twenty-seven dogs were classified into the CKD group. Their median age was 15 years (range, 5 to 19 years). Shih-tzu $(n=8)$ and Maltese terrier $(n=7)$ were the most common breeds, followed by miniature schnauzer $(n=2)$, mixed breed $(n=2)$, cocker spaniel $(n=1)$, golden retriever $(n=1)$, Japanese chin $(n=1)$, Pomeranian $(n=1)$, and Yorkshire terrier $(n=1)$. Twelve dogs were castrated males, three were intact males, nine were spayed females, and three were intact females. Dogs in the CKD group were classified into different stages according to IRIS CKD guidelines based on serum creatinine and sSDMA concentrations: (1) stage 1 (creatinine $<1.4 \mathrm{mg} / \mathrm{dL}$ and $\mathrm{sSDMA}<18 \mu \mathrm{g} / \mathrm{dL} ; n=8$ ); (2) stage 2 (creatinine 1.4 to $2.8 \mathrm{mg} / \mathrm{dL}$ or sSDMA 18 to $35 \mu \mathrm{g} / \mathrm{dL} ; n=8$ ); (3) stage 3 (creatinine 2.9 to $5.0 \mathrm{mg} / \mathrm{dL}$, sSDMA 36 to $54 \mu \mathrm{g} / \mathrm{dL} ; n=7$ ); (4) stage 4 (creatinine > $5.0 \mathrm{mg} / \mathrm{dL}$ or sSDMA $>54 \mu \mathrm{g} / \mathrm{dL} ; n=4)$. Dogs were classified into different substages based on the UPC: (1) nonproteinuric (UPC $<0.2 ; n=9$ ); (2) borderline proteinuric (UPC: 0.2 to $0.5 ; n=7$ ); and (3) proteinuric (UPC > $0.5 ; n=11)$. Dogs were also classified into substages based on systolic blood pressure (BP): (1) normotensive (BP < $140 \mathrm{mmHg} ; n=11$ ); (2) prehypertensive (BP: 141 to $159 \mathrm{mmHg} ; n=2$ ); (3) hypertensive (BP: 160 to $179 \mathrm{mmHg} ; n=9$ ); and (4) severely hypertensive (BP $\geq$ $180 \mathrm{mmHg} ; n=5)$. Ten dogs were included in the control group; the median age was 5 years and 6 months (range, 2 to 18 years). Maltese terrier $(n=4)$ and Pomeranian $(n=3)$ were the most common breeds. Other 
breeds included beagle $(n=2)$ and Jindo $(n=1)$. Three dogs were castrated males, one was an intact male, four were spayed females, and two were intact females. To identify the associations between soluble alpha-klotho concentration and age, dogs in both control and CKD groups were classified into different groups based on age: (1) same or under 2 years $(n=3)$; (2) 3 to 8 years $(n=10)$; (3) 9 to 15 years $(n=12)$; and (4) 16 years or over $(n=12)$.

Dogs tentatively diagnosed with acute kidney injury or tumors in the urinary tracts were excluded. The study was conducted in animals with full consent from their owners and any treatments needed were not withheld. This study was approved by the Institutional Animal Care and Use Committee of Chungnam National University. After the sampling procedure, dogs were taken care of by their owners as usual.

\section{Sampling and analysis}

All dogs underwent a complete physical examination, including measurement of blood pressure using a Doppler device. Blood samples were taken from the jugular vein. Within 15 minutes after collection, samples were centrifuged at $8000 \mathrm{rpm}$ for 15 minutes. After measurement of blood urea nitrogen, creatinine, and inorganic phosphorus concentrations using a BS-200 analyzer (Mindray, Shenzhen, China), the remaining serum samples were frozen at $-80{ }^{\circ} \mathrm{C}$. Urine samples were collected by either cystocentesis or catheterization after blood samples were collected. After routine urinalysis, samples were centrifuged at $3000 \mathrm{rpm}$ for 20 minutes. The supernatant was frozen at $-80{ }^{\circ} \mathrm{C}$.

Serum and urinary alpha klotho concentrations were measured using a commercially available canine-specific sandwich enzyme-linked immunosorbent assay (ELISA) kit (MyBioSource, San Diego, USA). This quantitative sandwich ELISA is based on soluble alpha klotho antibody-antigen interactions and an HRP colorimetric detection system to detect soluble alpha klotho antigen targets in samples. Samples were thawed at room temperature before klotho and SSDMA analysis. Serum SDMA level was measured with a Catalyst $\mathrm{ONE}^{\circ}$ (IDEXX, Westbrook, USA), which is an automated analyzer that analyzes dry slides with a reactive reagent based on ELISA. BUN, creatine, and phosphorus were measured with a BS-200 (Mindray, Shenzen, China), which is an automated chemistry analyzer that uses a photometric system of 8 monochromatic lights to measure the absorbance of the reaction liquid in rotating reaction cuvettes. The urine protein-to-creatinine ratio was measured with a VetTest $8008^{\circ}$ (IDEXX, Westbrook, USA) using thawed urine samples when ELISA was performed.

\section{Statistical analysis}

The measured parameters are provided as median value and range. The Kolmogorov-Smirnov test was used to analyze the normality of the distribution of parameters. $\mathrm{UrKl} / \mathrm{Cr}$ and serum alpha klotho concentrations were not normally distributed. Therefore, $\mathrm{UrKl} / \mathrm{Cr}$ and serum alpha klotho concentrations were compared between groups by a nonparametric Kruskal-Wallis test. The Mann-Whitney U-test for nonparametric data was used for confirmation of differences between groups. Spearman's correlation coefficient was used to evaluate the relationships between variables. A $p$ value of $<0.05$ was considered statistically significant. A stepwise multiple regression analysis was performed with sSDMA, BUN, creatinine and phosphorus concentrations to estimate the effects of independent predictors on klotho concentrations. All analyses were performed using IBM SPSS Statistics 24 (IBM, New York, USA).

\section{Abbreviations \\ BUN: Blood urea nitrogen; CKD: Chronic kidney disease; CKD-MBD: Chronic kidney disease-mineral bone disorder; ELISA: Enzyme-linked immunosorbent assay; FGF: Fibroblast growth factor; eGFR: Estimated glomerular filtration rate; GFR: Glomerular filtration rate; IP-IB: Immunoprecipitation-immunoblot; IRIS: International Renal Interest Society; RSHP: Renal secondary hyperparathyroidism; sSDMA: Serum symmetric dimethylarginine; UrKI/ Cr: Urine klotho-to-creatinine ratio; UPC: Urine protein-to-creatinine ratio}

\section{Acknowledgments}

This manuscript is written based on the Master's thesis of author Hong jae Yi. This study was supported by "Cooperative Research Program of Center for Companion Animal Research (Project No. PJ01404502): Rural Development Administration, Republic of Korea. The authors wish to thank Korea animal medical center (Cheongju-si, Chungcheongbuk-do, Republic of Korea) and Africa animal medical center (Daejeon-si, Chungcheongbuk-do, Republic of Korea) for providing sites for sampling.

\section{Authors' contributions}

$\mathrm{HJ}, \mathrm{KW}$, and KP designed the research. $\mathrm{HJ}$ collected and analyzed samples. $\mathrm{HJ}$ wrote the manuscript. JB, KP, Yl and $\mathrm{KH}$ contributed to analysis and interpretation of the data and revised the manuscript. All authors read and approved the final manuscript.

\section{Funding}

Not applicable

\section{Availability of data and materials}

The datasets generated during and/or analyzed during the current study are available from the corresponding author on reasonable request.

\section{Ethics approval and consent to participate}

This study was approved by the Institutional Animal Care and Use Committee of Chungnam National University (Approval number: 201906ACNU-072). This study was conducted in animals with full written consent from their owners.

\section{Consent for publication}

Not applicable.

\section{Competing interests}

The authors declare that they have no competing interests.

\section{Author details}

${ }^{1}$ VMTH of College of Veterinary Medicine, Chungnam National University, N13-2, \#308, 99 Daehak-ro, Yuseong-gu, Daejeon, Republic of Korea. ${ }^{2}$ College of Veterinary Medicine, Kangwon National University, Chuncheon, Korea. 
Received: 28 February 2020 Accepted: 6 July 2020 Published online: 16 July 2020

\section{References}

1. Brown S. Management of chronic kidney disease. 2 ed. Grauer JEG, editor: British Small Animal Veterinary Association Gloucester; 2007.

2. Cortadellas O, Fernandez del Palacio MJ, Talavera J, Bayon A. Calcium and phosphorus homeostasis in dogs with spontaneous chronic kidney disease at different stages of severity. J Vet Intern Med. 2010;24(1):73-9.

3. IRIS. IRIS staging of CKD: International Renal Interest Society; 2019 [Available from: http://www.iris-kidney.com/guidelines/staging.html.

4. Silver J, Naveh-Many T. FGF-23 and secondary hyperparathyroidism in chronic kidney disease. Nat Rev Nephrol. 2013;9(11):641-9.

5. Zou D, Wu W, He Y, Ma S, Gao J. The role of klotho in chronic kidney disease. BMC Nephrol. 2018;19(1):285.

6. Foster JD. Update on Mineral and Bone Disorders in Chronic Kidney Disease. Vet Clin North Am Small Anim Pract. 2016;46(6):1131-49.

7. Hu MC, Kuro-o M, Moe OW. The emerging role of Klotho in clinical nephrology. Nephrol Dial Transplant. 2012;27(7):2650-7.

8. Salanova Villanueva L, Sanchez Gonzalez C, Sanchez Tomero JA, Aguilera A, Ortega Junco E. Bone mineral disorder in chronic kidney disease: Klotho and FGF23; cardiovascular implications. Nefrologia. 2016;36(4):368-75.

9. Takenaka T, Inoue T, Miyazaki T, Hayashi M, Suzuki H. Xeno-Klotho Inhibits Parathyroid Hormone Signaling. J Bone Miner Res. 2016:31(2):455-62.

10. Kuro OM. The FGF23 and Klotho system beyond mineral metabolism. Clin Exp Nephrol. 2017;21(Suppl 1):64-9.

11. Yamada S, Giachelli CM. Vascular calcification in CKD-MBD: Roles for phosphate, FGF23, and Klotho. Bone. 2017;100:87-93.

12. Kuro-o M, Matsumura $Y$, Aizawa $H$, Kawaguchi $H$, Suga $T$, Utsugi T, et al. Mutation of the mouse klotho gene leads to a syndrome resembling ageing. Nature. 1997;390(6655):45-51.

13. Hu MC, Shi M, Zhang J, Pastor J, Nakatani T, Lanske B, et al. Klotho: a novel phosphaturic substance acting as an autocrine enzyme in the renal proximal tubule. FASEB J. 2010;24(9):3438-50.

14. Kato Y, Arakawa E, Kinoshita S, Shirai A, Furuya A, Yamano K, et al. Establishment of the anti-Klotho monoclonal antibodies and detection of Klotho protein in kidneys. Biochem Biophys Res Commun. 2000;267(2):597602.

15. Kurosu H, Ogawa Y, Miyoshi M, Yamamoto M, Nandi A, Rosenblatt KP, et al. Regulation of fibroblast growth factor-23 signaling by klotho. J Biol Chem. 2006:281(10):6120-3.

16. Urakawa I, Yamazaki Y, Shimada T, lijima K, Hasegawa H, Okawa K, et al. Klotho converts canonical FGF receptor into a specific receptor for FGF23. Nature. 2006;444(7120):770-4

17. Medici D, Razzaque MS, Deluca S, Rector TL, Hou B, Kang K, et al. FGF-23Klotho signaling stimulates proliferation and prevents vitamin D-induced apoptosis. J Cell Biol. 2008;182(3):459-65.

18. Yoshida T, Fujimori T, Nabeshima Y. Mediation of unusually high concentrations of 1,25-dihydroxyvitamin D in homozygous klotho mutant mice by increased expression of renal 1alpha-hydroxylase gene. Endocrinology. 2002;143(2):683-9

19. Shimada T, Kakitani M, Yamazaki Y, Hasegawa H, Takeuchi Y, Fujita T, et al. Targeted ablation of Fgf23 demonstrates an essential physiological role of FGF23 in phosphate and vitamin D metabolism. J Clin Invest. 2004;113(4): 561-8.

20. Murali SK, Roschger P, Zeitz U, Klaushofer K, Andrukhova O, Erben RG FGF23 Regulates Bone Mineralization in a 1,25(OH)2 D3 and KlothoIndependent Manner. J Bone Miner Res. 2016;31(1):129-42.

21. Bloch $L$, Sineshchekova O, Reichenbach D, Reiss K, Saftig P, Kuro-o M, et al. Klotho is a substrate for alpha-, beta- and gamma-secretase. FEBS Lett. 2009; 583(19):3221-4.

22. Chen CD, Podvin S, Gillespie E, Leeman SE, Abraham CR. Insulin stimulates the cleavage and release of the extracellular domain of Klotho by ADAM10 and ADAM17. Proc Natl Acad Sci U S A. 2007;104(50):19796-801.

23. Chen CD, Tung TY, Liang J, Zeldich E, Tucker Zhou TB, Turk BE, et al. Identification of cleavage sites leading to the shed form of the anti-aging protein klotho. Biochemistry. 2014;53(34):5579-87.

24. Hu MC, Shi M, Zhang J, Addo T, Cho HJ, Barker SL, et al. Renal Production, Uptake, and Handling of Circulating alphaKlotho. J Am Soc Nephrol. 2016; 27(1):79-90.
25. Hu MC, Shi M, Gillings N, Flores B, Takahashi M, Kuro OM, et al. Recombinant alpha-Klotho may be prophylactic and therapeutic for acute to chronic kidney disease progression and uremic cardiomyopathy. Kidney Int. 2017:91(5):1104-14.

26. Kurosu H, Yamamoto M, Clark JD, Pastor JV, Nandi A, Gurnani P, et al. Suppression of aging in mice by the hormone Klotho. Science. 2005; 309(5742):1829-33.

27. Akimoto T, Yoshizawa H, Watanabe $Y$, Numata A, Yamazaki T, Takeshima E, et al. Characteristics of urinary and serum soluble Klotho protein in patients with different degrees of chronic kidney disease. BMC Nephrol. 2012;13:155.

28. Imura A, Iwano A, Tohyama O, Tsuji Y, Nozaki K, Hashimoto N, et al. Secreted Klotho protein in sera and CSF: implication for post-translational cleavage in release of Klotho protein from cell membrane. FEBS Lett. 2004; 565(1-3):143-7.

29. Semba RD, Moghekar AR, Hu J, Sun K, Turner R, Ferrucci L, et al. Klotho in the cerebrospinal fluid of adults with and without Alzheimer's disease. Neurosci Lett. 2014;558:37-40.

30. Akimoto T, Kimura T, Watanabe Y, Ishikawa N, Iwazu Y, Saito O, et al. The impact of nephrectomy and renal transplantation on serum levels of soluble Klotho protein. Transplant Proc. 2013;45(1):134-6. .

31. Lindberg K, Amin R, Moe OW, Hu MC, Erben RG, Ostman Wernerson A, et al. The kidney is the principal organ mediating klotho effects. J Am Soc Nephrol. 2014;25(10):2169-75.

32. Asai O, Nakatani K, Tanaka T, Sakan H, Imura A, Yoshimoto S, et al. Decreased renal alpha-Klotho expression in early diabetic nephropathy in humans and mice and its possible role in urinary calcium excretion. Kidney Int. 2012;81(6):539-47.

33. Shimamura $Y$, Hamada K, Inoue $K$, Ogata K, Ishihara M, Kagawa T, et al. Serum levels of soluble secreted alpha-Klotho are decreased in the early stages of chronic kidney disease, making it a probable novel biomarker for early diagnosis. Clin Exp Nephrol. 2012;16(5):722-9.

34. Pavik I, Jaeger P, Ebner L, Wagner CA, Petzold K, Spichtig D, et al. Secreted Klotho and FGF23 in chronic kidney disease Stage 1 to 5: a sequence suggested from a cross-sectional study. Nephrol Dial Transplant. 2013;28(2): 352-9.

35. Devaraj S, Syed B, Chien A, Jialal I. Validation of an immunoassay for soluble Klotho protein: decreased levels in diabetes and increased levels in chronic kidney disease. Am J Clin Pathol. 2012;137(3):479-85.

36. Kim HR, Nam BY, Kim DW, Kang MW, Han JH, Lee MJ, et al. Circulating alpha-klotho levels in CKD and relationship to progression. Am J Kidney Dis. 2013;61(6):899-909.

37. Seiler S, Wen M, Roth HJ, Fehrenz M, Flugge F, Herath E, et al. Plasma Klotho is not related to kidney function and does not predict adverse outcome in patients with chronic kidney disease. Kidney Int. 2013;83(1):121-8.

38. Harjes LM, Parker VJ, Dembek K, Young GS, Giovaninni LH, Kogika MM, et al. Fibroblast Growth Factor-23 Concentration in Dogs with Chronic Kidney Disease. J Vet Intern Med. 2017;31(3):784-90

39. $X u Y$ Y, Sun Z. Molecular basis of Klotho: from gene to function in aging. Endocr Rev. 2015;36(2):174-93.

40. Barker SL, Pastor J, Carranza D, Quinones H, Griffith C, Goetz R, et al. The demonstration of alphaKlotho deficiency in human chronic kidney disease with a novel synthetic antibody. Nephrol Dial Transplant. 2015;30(2):223-33.

41. Neyra JA, Hu MC. Potential application of klotho in human chronic kidney disease. Bone. 2017;100:41-9.

42. Heijboer AC, Blankenstein MA, Hoenderop J, de Borst MH, Vervloet MG, Consortium N. Laboratory aspects of circulating alpha-Klotho. Nephrol Dial Transplant. 2013;28(9):2283-7.

43. Neyra JA, Moe OW, Pastor J, Gianella F, Sidhu SS, Sarnak MJ, et al. Performance of soluble Klotho assays in clinical samples of kidney disease. Clinical Kidney Journal. 2019

44. Yamazaki Y, Imura A, Urakawa I, Shimada T, Murakami J, Aono Y, et al. Establishment of sandwich ELISA for soluble alpha-Klotho measurement: Age-dependent change of soluble alpha-Klotho levels in healthy subjects. Biochem Biophys Res Commun. 2010;398(3):513-8.

\section{Publisher's Note}

Springer Nature remains neutral with regard to jurisdictional claims in published maps and institutional affiliations. 